\title{
Algumas Aplicações Analíticas dos Ultra-sons
}

MAURO KORN ${ }^{1}$, MADSON DE GODOI PEREIRA ${ }^{1}$, SIVANILDO DA SILVA BORGES²

\section{Resumo}

Alguns dos efeitos químicos produzidos pelos ultra-sons (transformaçōes) são conhecidos desde os primeiros anos do século passado. Apesar disso, os químicos analíticos têm empregado a técnica de sonicação como uma opção para agitação e para auxiliar em processos de extração e/ou lixiviação. Contudo, normalmente há uma forte tendência em abdicar de efeitos peculiares e inerentes à irradiação de soluçōes com ondas ultra-sônicas de baixa frequência. Ao não relevar os possíveis efeitos químicos resultantes da sonicação (cavitação acústica), as características originais das amostras irradiadas poderão ser irreversivelmente comprometidas, visto que es- pécies químicas sob investigação podem ser geradas no meio irradiado, enquanto que analitos poderão ser transformados ou eliminados das soluções.

Ao deter conhecimento sobre o que pode ser transformado, como tais transformações ocorrem e sobre os produtos desta transformação, uma gama de possibilidades de aplicações analíticas com processos assistidos por ultra-sons de baixa frequência será explorada. Nesta perspectiva, serão abordadas aplicaçōes dos ultra-sons na decomposição de resíduos orgânicos, geração in situ de reagentes e mediação de procedimentos alternativos de análise química.

\section{Introdução}

Ultra-sons sẫo ondas mecânicas que se propagam através de qualquer meio material com freqüência maior que 20 $\mathrm{kHz}$. O primeiro gerador de ondas ultra-sônicas foi reportado na literatura em 1900, em artigo publicado pelo Prof. Edelmann, no qual foram descritos os detalhes da construção do tubo de Galton. Nas figuras la e b podem ser observadas a primeira página do artigo do Prof. Edelmann (a) e um exemplar de um tubó de Gâlton (b), datådos dé 1900.

Os ultra-sons respondem por uma região do espectro acústico, o qual é subdividido em três regiões principais - a faixa do infra-sons $(v<20 \mathrm{~Hz})$; a faixa sons ( $20 \mathrm{~Hz}<v<20 \mathrm{kHz}$ ), perceptiveis aos humanos; e, a faixa dos ultra-sons ( $v>20 \mathrm{kHz}$ ). Para os ultra-sons pode ser assumida, ainda, uma divisão de faixas: a dos ultra-sons de baixa freqüên- cia $(20 \mathrm{kHz}<v<1 \mathrm{MHz})$ e de alta frequềncia $(v>1 \mathrm{MHz})$. As ondas ultra-sônicas de baixas freqüências são aquelas que poodem apresentantar altá potênciá, enquanto que as ondas ultra-sônicas de altả frequêểncia tềm baixả potềnciá, podendo ser aplicadas principalmente para fins de diagnóstico seja na medicina como na engenharia. Desta forma, aqui iremos discutir exclusivamente as ações de ondas ultra-sônicas de baixa freqüência, as quais levam a alterações químicas e físicas no meio líquido onde as ondàs são aplicadàs.

Geradores de ondas ultra-sônicas de baixa freqüência são comumente empregados em laboratórios de química e de biologia, seja para a limpeza de materiais, seja para o rompimento das paredes celulares. Os equipamentos usualmente empregados são o banho e a sonda ultra-sônica.
Poucos são os laboratórios químicos que não têm um banho ultra-sônico de limpeza. Estes banhos são produzidos e comercializados por grande variedade de empresas, sendo que estes equipamentos são fabricados pelo acoplamento de um ou mais cristais piezeletricos na parte inferior de um vaso metálico, preferencialmente construído em aço. Aplicada uma dada diferença de potencial nas faces laterais de um transdutor piezelétrico, serão provocadas vibraçốes nas faces perpendiculares do dispositivo e está vibrarả a ưná frequeuência prèdeterminada. Banhos ultra-sônicos, com freqüência de 20 e 40 kHz, são os mais comumente empregados. Deve ser salientado que outros processos levam à geração de ondas ultra-sônicas, como o efeito magneto-estritivo, mas os equipamentos que fazem uso de transdutores piezelétricos săo mais comuns.

Quando líquidos são submetidos às ondas ultra-sônicas de alta potência,

\footnotetext{
${ }^{1}$ Laboratório de SonoFIA / DCET - Universidade do Estado da Bahia, R. Silveira Martins 2555, 41195-001, Salvador-BA, Brasil, Mauro Korn é Professor Titular de Química Analítica do Departamento de Ciências Exatas e da Terra da UNEB (mkorn@uneb.br)

${ }^{2}$ Instituto de Química - Universidàde Federă da Băhiả, Campus Universitário de Ondina, 40170-290, Salvador-BA, Brasil
} 
figura 1 Primórdios dos ultra-sons. (a) Artigo sobre a construçẫo do sonicador de Galton. (b) Exemplar de gerador de uitra-som de Galton. datado de 1900 . Figuras do acervo do Museu da Universidade de Innsbruck, Áustria. (http//info uibk ac at $/ \mathrm{c} / \mathrm{c} / \mathrm{c} 704 / \mathrm{museum} / \mathrm{en} / \mathrm{s}$ tarthtml)
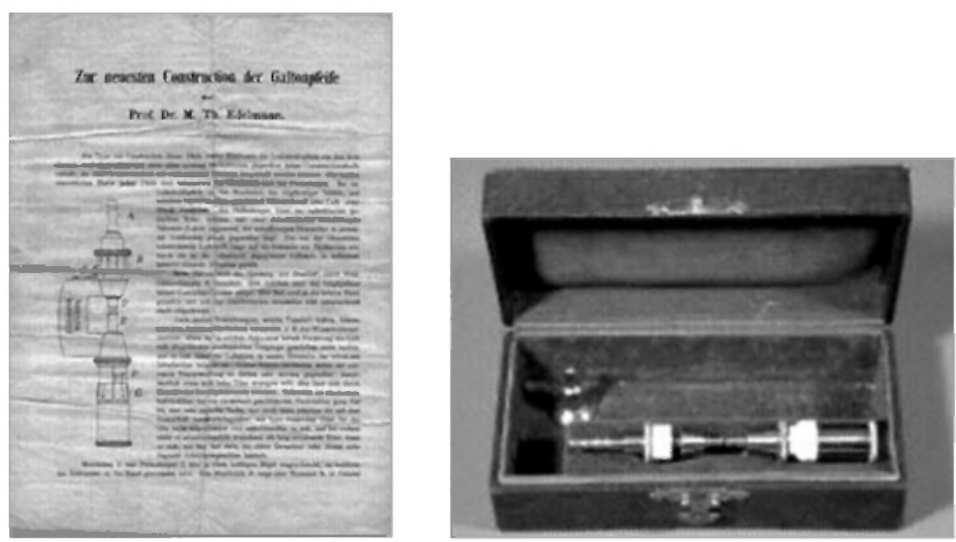

estas produzem intensas e sucessivas ondas de compressão e rarefação no meio, no qual, a depender da viscosidade, pode ocorrer o surgimento de cavidades de dimensões microscópicas durante uma fase de rarefação. A ocorrência de gases e vapores no meio irradiado faz com que moléculas dos gases e vapores migrem para o interior das cavidades. Nos sucessivos ciclos de compressão e rarefação as dimensões da cavidade vão aumentando (figura 2), até que seja atingido um diâmetro crítico, quando esta finalmente sofre colapso

Ondas ultra-sônicas de freqüência de $20 \mathrm{kHz}$ e intensidade de $1 \mathrm{~W} \mathrm{~cm}^{-2}$ ao se propagarem pela água (velocidade do som de $\sim 1500 \mathrm{~m} \mathrm{~s}^{-1}$ e densidade $=1 \mathrm{~g}$ $\mathrm{cm}^{-3}$ ), levam um corpúsculo a deslocamentos de $\sim 0,93 \mathrm{~mm}$, em torno de um ponto central, com velocidade máxima de 11,7 $\mathrm{m} \mathrm{s}^{-1}$. A aceleração máxima que este corpúsculo estaria submetido seria de $\sim 16 \mathrm{~km} \mathrm{~s}^{-2}$, ou seja, 1600 vezes maior que a acéleração da gravidade. Adicionalmente, a variação de pressão calculada para a situaçăo apresentada neste exemplo seria de 1,7 MPa. Porém este exemplo trata do efeito da propagaçãó dê ondá ultrā-sônića de $1 \mathrm{~W} \mathrm{~cm} \mathrm{~cm}^{2}$. Uma vez que processadores ultra-sônicos com potência superior a $100 \mathrm{~W}$ são facilmente encontrados, as velocidades maxximas, presssões e acelerações sẹão tão mais intensas quanto maior a potêncla do gerador de ultra-sons.

Os resultados obtidos em diferentes ensaios realizados com sistemas heterogêneos (líquido-sólido) sob sonicação indicaram que os efeitos dessas ondas acústicas não deveriam ser considerados de forma simplista, ou seja, como um mero e eficaz sistema de agitação. Os efeitos da sonicação nos sistemas heterogêneos sólido-líquido devem ser relacionados aos jatos de alta velocidade produzidos pela cavitação acústica nas proximidades das superfícies dos metais. Essas ondas mecânicas, ao interagirem com a superfície de sólidos, levam a alterações significativas na morfologia da superfície de sólidos. Caso os ultra-sons sejam aplicados em líquidos contendo material particulado em suspensão, as cavidades deixam de ser simétricas de forma que, quando de seu colapso, os microjatos são direcionados para o sólido.

Porém, a energia resultante da ação diretã dơs ultrā-sons não seriaa cảpãz dẹ provocar efeitos como aqueles observados durante a sonicação, como o rompimento de ligaçôes inter e intramoleculares. Estudos realizados com líquidos puros e soluções aquosas demonstraram outras potencialidades da aplicação da energía acústica, ao mesmo tempo em que induziram a uma reflexão sobre o emprego de ultra-som no preparo de amostras. Estas considerações estão relacionadas com os resultados obtidos nós primeiros estudós soobre à açăo de ondas ultra-sônicas em sistemas homogêneos realizados por Miller em 1950. Esses estudos que comprovaram a cisão das ligações $\mathrm{H}-\mathrm{O}$ em moléculas de água e a produçăo de peróxido de hidrogênio no meio irradiado devem ser considerados como os pioneiros na caracterização dos efeitos químicos provocados por ondas ultra-sônicas, visto que implicam na transformaçăo de espécies químicas. O fenômeno envolvido na cisăo homolítica de ligações foi denominado de sono- lise da água, o qual leva à produção direta dos radicais livres $\mathrm{He} \mathrm{HO}$ no meio.

A elevada reatividade dos radicais livres favorece a rápida interação destas espécies com íns, moléculas, ou ainda, a combinação entre radicais, com a consequente produção de novas espécies moleculares $\left(\mathrm{H}_{2} \mathrm{O}_{2}\right)$, ou ainda, novos radicais livres $\left(\mathrm{O}_{2} \mathrm{H}\right)$. Diferentes estudos constataram o efeito das ondas ultra-sônicas na oxidação de íons $\mathrm{Fe}^{2+}$ em meio aquoso, na degradação de polímeros e na formação de peróxido de hidrogênio, quando água pura foi submetida aos ultra-sons de baixa frequência. Também deve ser mencionado que em investigações recentes pôde ser constatada a diminuição significativa da taxa de formação de peróxido de hidrogênio sob sonicação com o aumento da concentração de $\mathrm{NaCl}$ presente na solução submetida a sonicação. Contudo, esses efeitos provocados pelo ultra-som não são habitualmente aplicados no desenvolvimento de métodos e procedimentós de análise química.

O Prof. Mason, da Faculdade de Ciências Aplicadas do Instituto Politécnico de Coventry (Inglaterra), no livro Chemistry with Ultrasound, fez importantes considerações sobre a influência dos ultra-sons na reatividade química. o autor enumerou alguns procedimentos gerais que proporcionam aumento da reatividade e estes, incluindo a irradiação ultra-sônica (Tabela 1). Segundo Mason, a inserção dos ultra-sons na lista de fatores que aumentam a reatividade é devido às evidências acumuladas sobre os efeitos provocados por estas ondas em sistemas químicos. A principal causa desses efeitos é a cavitação 


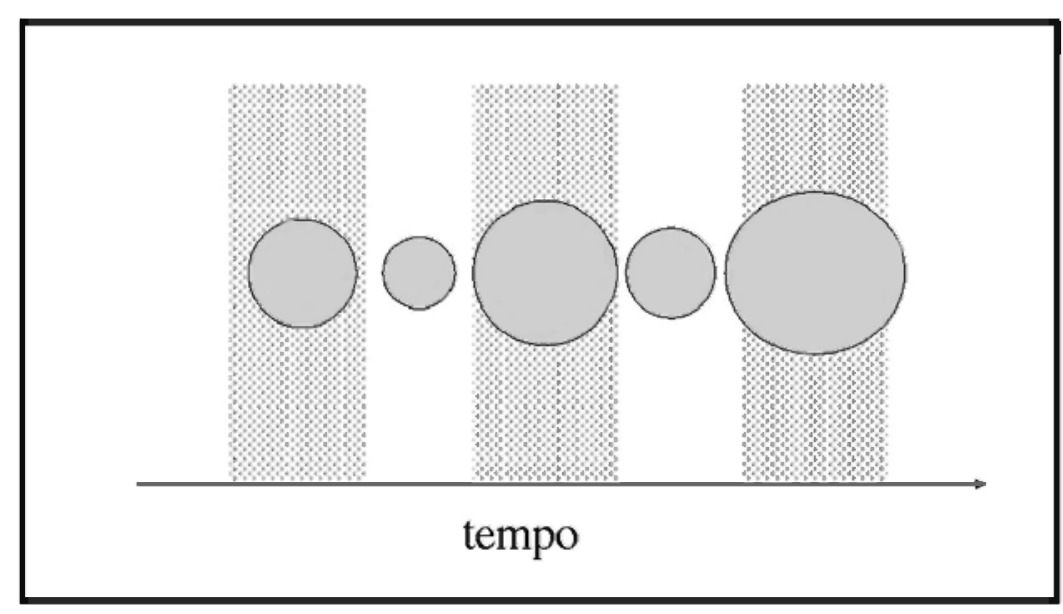

que pode ser considerada como o ciclo de formação, crescimento e colapso de bolhas micrométricas durante a sonicação. Com os colapsos das bolhas de cavitação ocorre a liberação de grande quantidade de energia para o meio, proporcionando na microrregião onde ocorreu o colapso, aumento da temperatura da ordem de alguns milhares de graus centígrados e da pressão para centenas de atmosferas.
Para fins analíticos os ultra-sons têm sido empregados em diferentes etapas, como apresentado na Tabela 2. Os usos mais corriqueiros dos ultra-sons em laboratórios de análise química são para limpeza de material e desgaseificação de soluções. Laboratórios que fazem uso da técnica de cromatografia a líquido empregam os banhos ultra-sônicos para a desgaseificação de soluções, como uma alternativa ao borbulhamento de $\mathrm{He}$, levando à redução dos custos

Tabela 1. Fatores que levam ao aumento da reatlvldade.

Aumento da temperatura de reação

Aumento da concentração de um reagente

Aumento da pressão aplicada no sistema

Emprego de catalisadores

Irradiação do sistema com ultra-som de alta potência

Tabela 2. Aplicações dos ultrà-sons nas diferentes etapas dả análise química

\begin{tabular}{ll}
\hline Etapas & Aplicações \\
\hline Amostragem & Não se Aplica \\
\hline Preparo da Amostra & Extração Sólido - Líquido \\
& Extração Líquido - Líquido \\
& Separação Granulométrica \\
& Desgaseificação \\
& Degradação de Matéria Orgânica \\
& Co-precipitação \\
& Geração de Reagentes \\
\hline Medida & Tempo de Propagação da Onda \\
& Atenuação da Intensidade da Onda \\
\hline Tratamento de Dados & Não se aplica \\
\hline
\end{tabular}

figura 2 A cavitaç̃o acústica

operacionais. Para este fim, a potência ultra-sônica deve ser suficientemente baixa, permitindo a produção e evolução de bolhas contendo gases dissolvidos na solução, sem que estas colapsem.

\section{Ultra-sons no Preparo de Amostras e Tratamento de Efluentes}

A energia liberada durante a cavitação acústica fornece excelentes perspectivas para o preparo e/ou tratamento de amostras e para a descontaminação de efluentes. No primeiro caso, o colapso das microbolhas favorece a extração de espécies químicas a partir de materiais sólidos, bem como a dissolução destes [1]; enquanto que, no segundo caso, as elevadas temperaturas e pressões proporcionam a degradação térmica e/ou radicalar de espécies orgânicas contaminantes [2], levando à completa mineralização das espécies para tempos prolongados de sonicaçăo [3].

As modificações físicas e químicas resultantes da cavitação acústica têm impulsionado novas estratégias de preparo de amostras [4-8] e, em sistemas heterogêneos, o tratamento é favorecido devido aos seguintes fenômenos [9-12]

- produção de emulsão nas interfaces de sistemas líquido-líquido;

- lixiviação na superfície em sistemas sólido-líquido;

- erosão, fragmentação e aumento da área superficial de partículas sólidas em decorrência das ondas de choque originadas da implosão das microboIhas; 
- diminuição do gradiente de concentração pelo aumento do transporte de massa ocasionado pela turbulência e microjatos.

A redução do tamanho de partícula configura-se como um dos mais notórios benefícios da sonicação nos procedimentos de lixiviação e dissolução, visto que muitas reações são limitadas pela área superficial efetiva exposta aos reagentes, resultando em perdas de reprodutibilidade, rendimento e produtividade analítica [13]. A ruptura das partículas - levando ao aumento da área superficial - é provocada pelo colapso de microbolhas nos interstícios do sólido ou pelos violentos choques entre partículas, que chegam a alcançar velocidades $>500 \mathrm{~m} \mathrm{~s}^{-1}$, promovendo até a fusão de fragmentos metálicos [14]. Por outro lado, bolhas formadas nas proximidades das partículas tornam-se assimétricas e, ao implodirem, originam microjatos de líquidos com velocidades $>100 \mathrm{~m} \mathrm{~s}^{-1}$ [10]. Estes jatos são sempre direcionados para a superfície do sólido, resultando na erosão e, conseqüentemente, na renovação da superfície, aumentando a reatividade entre solvente e amostra $[1,2]$. A Tabela 3 lista algumas publicações sobre a utilização do ultra-som em etapas de preparo de amostras, incluindo dissolução total e extraçăo de espécies orgânicas e inorgânicas.

A principal vantagem do ultra-som em extrações reside na diminuição do tempo de processamento e na utilização de soluções diluídas de ácidos minerais. Apesarar da eficiência de extração de ións metálicos ser dependente da matriz, natureza e concentraçăo da espécie extratora [15]; eventuais modificaçôes na forma original do ín metálico não são consideradas, caso as determinações sejam conduzidas para a obtenção da quantidade total do analito.

Pérez-Cid et al. [16] empregaram o ultra-som para extrair seqüencialmente $\mathrm{Cu}, \mathrm{Cr}, \mathrm{Ni}, \mathrm{Pb}$ e $\mathrm{Zn}$ em diferentes fraçóes de lodo obtidas de estaçóes de tratamento de efluentes, conseguindo uma diminuição do tempo de extração de 51 h para 22 min. Ruiz-Jiménez et al. [5] obtiveram lixiviação quantitativa de Cd e $\mathrm{Pb}$ em tecido vegetal mediante a sonicação das amostras por 5 min na presen-

Tabela 3. Aplicações de ultra-sons para o preparo de amostras

\begin{tabular}{llc}
\hline Objetivo Analítico & Matriz & Referência \\
Decomposição & Amostras diversas & 21 \\
Extração de metais & Tecidos vegetais & 22 \\
Extração de compostos orgânicos & Tecidos vegetais & 23 \\
Dispersão & Suspensões & 17 \\
Extração de princípios ativos & Ervas & 24 \\
Extração de níquel & Minério de níquel & 25 \\
Extração de poluentes orgânicos & Sedimentos marinhos & 26 \\
Extração de poluentes orgânicos & Tecidos vegetais & 27 \\
Extração de fenóis & Solos & $28-30$ \\
Extração de ferro & Tecidos vegetais & 31 \\
Extração de compostos aromáticos & Solos e sedimentos & $32-35$ \\
Extração de metais & Tecidos vegetais & 36 \\
Extração de pentaclorofenol & Madeira & 37 \\
& &
\end{tabular}

ça de $\mathrm{HNO}_{3} 8 \%(\mathrm{~m} / \mathrm{v})$. Neste caso, utilizou-se um sistema equipado com câmara para extração em fluxo.

Entre as várias outras aplicações dos ultra-sons para a determinação de metais, destacam-se a formação de suspensões para análise direta em sólidos [17], descontaminação de recipientes para armazenamento de amostras [18] e favorecimento cinético de reações de complexação [19]. É importante ressaltar que, nas situaçõ̃es acima mencionadass, à integogridåde e identidåde dás èspécies de interesse foram mantidas seguindo o critério da rastreabilidade [20]. Contudo, a integridade e identidade das espécies de interesse não poderão ser garantidas, caso o analito sofra transformações aleatórias e múltiplas durante o processo de análise. Neste sentido, o preparo de amostras empregando ultra-som pode acarretar em transformações múltiplas e bruscas das espécies de interesse notadamente se estās forem volátēis e, conséquentemente, forem admitidas no interior dás cavidades durante a sonicação. Entre os compostos orgânicos voláteis que são degradados durante a sonicação, o grupo dos clopometanos deve ser destacado, levando à geração de $\mathrm{HCl}, \mathrm{HClO}$ e $\mathrm{CO}_{2}$ [3]. Na tabela 3 são apresentadas algumas aplicações dos ultra-sons no preparo de amostras.
No âmbito da proteção ambiental, a energia ultra-sônica apresenta efeitos interessantes para o tratamento de águas e de efluentes aquosos, visto que a mesma leva à destruição de microorganismos patogênicos e promove a degradação térmica e/ou radicalar de diferentes classes de poluentes. Especificamente para moléculas orgânicas, a eficiência de decomposição dependerá de propriedades físicas e químicas destas - solubilidade, pressão de vapor, massa molar, hidrofobicidade, entre outras - sendo as mais voláteis, apolares e de menor massa molar, aquelas que são preferencialmente admitidas e degradadas no momento do colapso das bolhas de cavitąăão, comó anteriormente déscrito. Os compostos hidrofílicos, de baixa pressăo de vapor e maior massa molar sofrerão, em maior escala, um processo de decomposição no seio da sôluçăó, prōnonovidá pélós rảdicáis próduzidos pela implosăo das cavidades [36]. A depender do poluente, a combinação da energia ultra-sonora com processos avançados de oxidação, tais como radiolise e ozonolise, origina procedimentos promissores [38].

Diversos artigos têm sido publicados para ilustrar o emprego de ultra-sons na degradação de tetracloreto de carbono em efluentes [39-41]; fenóis em amostras de águas [42-45]; corantes em efluentes industriais [46,47]; fenantreno 
[48] e hidrocarbonetos voláteis [49] em águas; além de compostos organoclorados [41, 50-54], organofluorados [55-57] e aromáticos [58-60] e explosivos [61] em águas.

Adicionalmente, a eficácia do ultra-som na descontaminaçăo de efluentes aquosos, quanto à presença de microorganismos, tem sido observada desde a primeira metade do século XX [62] e é atribuída à ruptura das células microbianas, ocasionando o escoamento do líquido citoplasmático [38].

\section{Considerações Finais}

A aplicação de formas não usuais de energia, como as oriundas de ultra-sons de baixa freqüência e alta potência, para assistir procedimentos de análise química devem ser consideradas como promissoras. A energia liberada durante a cavitação acústica fornece excelentes perspectivas para o preparo de amostras, bem como para algumas etapas acessórias ao processamento analítico, como podem ser considerados a geração de reagentes e o tratamento de resíduos de análises químicas.

Agradecimentos

MK e MGP agradecem ao apoio da Fundação de Amparo à Pesquisa do Estado da Bahia (FAPESB), a Secretaria de Ciência, Tecnologia e Inovação do Governo do Estado da Bahía (SECTI) e ao ConseIho Nacional de Desenvolvimento Científiço e Téchoológicó (MCT/CNPq) pèlō ãpōio às ătividades de pesquisá. MK e SSB agradecem à Coordenação de Aperfeiçoamento de Pessoal de Nível Superior do Ministério da Educação (MEC/CAPES).

Referências Bibliográficas

[1] Mason T. J., Lorimer J. P., Sonochemistry: Theory, Applications and Uses of Ultrasound in Chemistry, Ellis Horwood Limited, Chichester, UK, 1988.

[2] Suslick K. S.; Science 1990, 247, 1439.

[3] Francony, A.; Pétrier, C.; Ultrason. Sonochem. 1996, 3, 577

[4] Nascentes, C. C.; Korn, M.; Arruda, M. A. Z.; Microchem. J. 2001, 69, 37.
[5] Ruiz-Jiménez, J.; Luque-García, J. L.; Luque de Castro, M. D.; Anal. Chim. Acta 2003, 480, 231.

[6] Filgueiras, A. V.; Capelo, J. L.; Lavilla, I.; Bendicho, C.; Talanta 2000, 43, 433.

[7] Shiowatana, J.; Tantidanai, N.; Nookabkaew, S.; Nacapricha, D.; Environ. Int. 2001, 26, 381.

[8] Meegoda, J. N.; Perera, R.; J. Hazard. Mat. 2001, 85, 73.

[9] Ashley, K.; Trends Anal. Chem. 1998, 17, 366.

[10] Luque-García, J. L.; Luque de Castro, M. D.; Trends Anal. Chem. 2003, 22, 41.

[11] Pino, V.; Ayala, J. H.; Afonso, A. M.; González, V.; Talanta 2001, 54, 15.

[12] Babic, S.; Petrovic, M.; KastelanMacan, M.; J. Chromatogr. A 1998, 823.

[13] Krug, F. J., "Methods of Sample Decomposition", In: IV Workshop on Sample Preparation, Salvador-Brazil, 2003.

[14] Suslick, K. S.; Didenko, Y.; Fang, M. M. Hyeon, T.; Kolbeck, K. J.; McNamara, W. B. Mdleleni, M. M.; Wong, M.; Phil. Trans. R. Soc. Lond. A 1999, 357, 335

[15] Al-Merey, R.; Al-Masri, M. S.; Bozou, R.; Anal. Chim. Acta 2002, 452, 143.

[16] Pérez-Cid, B.; Lavilla, I.; Bendicho, C.; Anal. Chim. Acta 1998, 360, 35

[17] Santos, C.; Alava-Moreno, F.; Lavilla, I.; Bendicho C.;. Anal. Atom. Spectrom. 2000 $15,987$.

[18] Kinsella, B.; Willix, R. L.; Anal. Chem. 1982, 54, 2614.

[19] Patnaik, U.; Muralidhar, J.; Talanta 1995, 42, 553.

[20] Valcárcel, M. "Principles of Analytical Chemistry". Ed. Springer-Verlag, Berlin Heidelberg, 2000.

[21] Goncharova, N. N.; Bukharova, Y. A.; Kuznetsova, T. V.; Utenkova, T. I.; J. Anal. Chem. 1999, 54, 1094

[22] Arruda, S. C. C.; Rodriguez, A. P. M. Arruda, M. A. Z.; J. Braz. Chem. Soc. 2003 14, 470 .

[23] Mason, T. J.; Ultrason. Sonochem. 2000, 7, 145.

[24] Vinatoru, M.; Ultrason. Sonochem. 2001, 8, 303.

[25] Swamy, K. M.; Sukla, L. B.; Narayana, K. L.; Kar, R. N.; Panchanadikar, V. V.; Ultrason. Sonochem. 1995, 2, S5.

[26] Pastor, A.; Vazquez, E.; Ciscar, R.; De la Guardia, M.; Anal. Chim. Acta 1997, 344, 241.

[27] Camel, V.; Trends Anal. Chem. 2000, 19, 229.
[28] Llompart, M. P.; Lorenzo, R. A.; Cela, R.; Pare, J. R. J.; Analyst 1997, 122, 133.

[29] Llompart, M. P.; Lorenzo, R. A.; Cela, R.; Pare, J. R. J.; Belanger, J. M. R.; Li, K.; J. Chromatogr. A 1997, 757, 153.

[30] Llompart, M. P.; Lorenzo, R. A.; Cela, R.; Li, K.; Belanger, J. M. R.; J. Chromatogr. A 1997, 774, 243.

[31] Lázaro, F.; Luque de Castro, M. D.; Valcárcel, M.; Anal. Chim. Acta 1991, 242 , 283.

[32] Heekem, O. P.; Theobald, N.; Wenclawiak, B. W.; Anal. Chem. 1997, 69, 2171.

[33] Sun, E. S.; Littlejohn, D.; Gibson, M. D.; Anal. Chim. Acta 1998, 364, 1

[34] Marvin, C. H.; Allan, L.; McCarry, B. E. Bryant, D. W.; Int. J. Environ. Anal. Chem. 1992, 49, 221.

[35] Kayali-Sayadi, M. N.; Rubio-Barroso, S.; Díaz-Díaz, C. A.; Polo-Díez, L. M.; Fresenius J. Anal. Chem. 2000, 368, 697.

[36] Naffrechoux, E.; Chanoux, S.; Petrier, C.; Suptil, J.; Ultrason. Sonochem. 2000, 7, 255.

[37] Gogate, P. R.; Mujumdar, S.; Pandit, A B.; Advance in Environmental Research 2003, 7, 283.

[38] Tiehm, A.; Mason, T. J.; Advances in Sonochemistry. - Uitrasound in Environmental Protection - Vol. 6. New York: Elsevier Science B. V., 2001, 273p.

[39] Borges, S. S.; Korn, M.; Quim. Nova 2002, 25, 558 .

[40] Hung, H. M.; Hoffmann, M. R.; Environ Sci. Technor. 1998, 32, 3011.

[41] Huà, I.; Hoffinăhn, M. R.; Environ. Sci Technol. 1996, 30, 864.

[42] Hão, H.; Chen, Y,; Wu, M.; Wang, H.; Yin, Y.; Lu, Z.; Uitrason. Sonochem. 2004 $11,43$.

[43] Yim, B.; Yoo, Y.; Maeda, Ys; Chemosphere 2003, 50, 1015.

[44] Kotonarou, A.; Mills, G.; Hoffmann, M R.; J. Phys. Chem. 1991, 95, 3630.

[45] Pétrier, C.; Lamy, M. F.; Francony, A.; Benahcene, A.; David, B.; Renaudin, V.; Gondrexon, N.; J. Phys. Chem. 1994, 98, 10514.

[46] Lorimer, J. P.; Mason, T. J,; Plattes, M. Phull, S. S.; Utrason. Sonochem. 2000, 7 , 237.

[47] Vinodgopal, K.; Peller, J.; Makogon, O.; Kámat, P. V.; Wat. Rés. 1998, 32, 3646.

[48] Littlē, C.; Hêlpher, M. J.; El-Shărif, M.; Uitrasonics 2002, 40, 667.

[49] Cheung, H. M.; Bhatnagar, A.; Jansen G.; Environ. Sci. Technol. 1991, 25, 1510. 
[50] Orzechowska, G. E.; Poziomek, E. I.; Hodge, V. F.; Engelmann, W. H.; Environ. Sci. Technol. 1995, 29, 1373.

[51] Yim, B.; Okuno, H.; Nagata, Y.; Maeda, Y.; J. Hazardous Materials 2001, B81, 253.

[52] Drijvers, D.; van Langenhove, H.; Kim, L. N. T.; Bray, L.; Ultrason. Sonochem. 1999 , 6. 115

[53] Hung, H.; Hoffmann, M.; J. Phys Chem. 1999, A103, 2734.
[54] Inazu, K.; Nagata, Y.; Maeda, Y.; Chem. Lett. 1993, 57.

[55] Nagata, Y.; Hirai, K.; Okitus, K.; Maeda, Y.; Chem. Lett. 1995, 203.

[56] Hirai, K.; Nagata, Y.; Maeda, Y.; Uitrason. Sonochem. 1996, 3, S205.

[57] Cheung, H. M.; Kurup, S.; Environ. Sci. Technol. 1994, 28, 1619.

[58] Pétrier, C.; Jiang, Y.; Lamy, M. F.; Environ. Sci. Technol. 1998, 32, 1316.
[59] Weavers, L. K.; Ling, F. H.; Hoffmann, M. R.; Environ. Sci. Technol. 1998, 32 2727.

[60] Visscher, A.; Eenoo, P.; Drijvers, D. Langenhove, H.; J. Phys. Chem. 1996, 100 11636.

[61] Qadir, L. R.; Osburn-Atkison, E. J.; Swider-Lyons, K. E.; Cepak, V. M.; Robinson, D. R.; Chemosphere 2003, 50, 1107.

[62] Mason, T. J.; Joyce, E.; Phull, S. S.; Lorimer, J. P.; Ultrason. Sonochem. 2003, 10, 319.
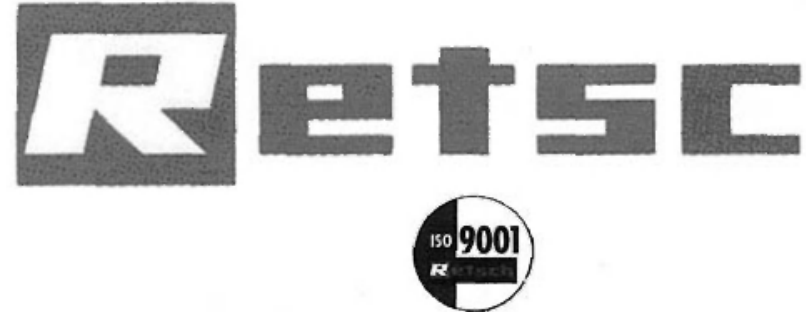

Moinhos

Agitadores de peneiros/peneiros
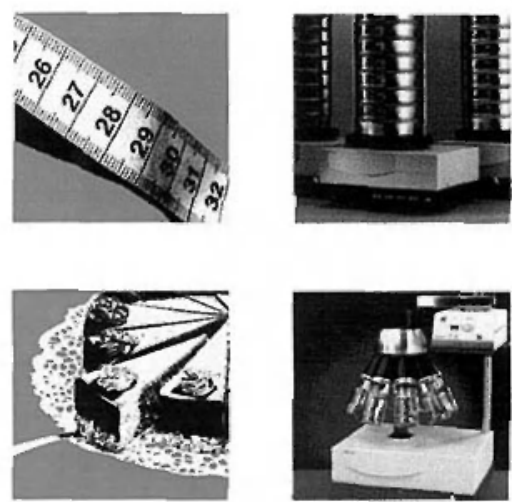

\section{Análise granulométrica automática CAMSIZER / CrYSTALSIZER}

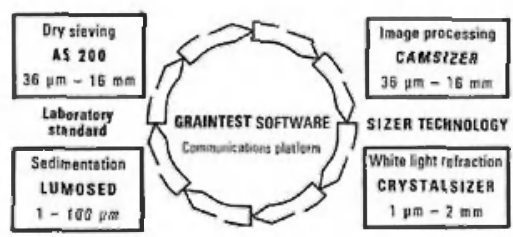

Peça-nos o contacto do agente mais próximo, através dos telefones:

$$
\text { 21-352 } 7293
$$

LISBOA

Campo Mártires da Pátria, 109 Raa do Vilarinho, $1235 \cdot 4100-517$, Port http://www.en-equipamentos.pt
22-618 4232

O Impartadar Exclusiva 